\title{
$7 \mathrm{~cm}$ großes Hirnaneurysma quetschte Sehnerv
}

\author{
US-Ärzte trauten ihren Augen \\ kaum: Bei einem Patienten mit \\ Sehstörungen und kognitiven \\ Problemen fanden sie ein $70 \mathrm{~mm}$ \\ großes Hirnaneurysma.
}

Der 55-jährige Mann hatte schon seit drei Jahren ein Sehproblem mit dem rechten Auge. In dieser Zeit hatten auch seine geistigen Fähigkeiten gelitten, und er entwickelte zunehmend Persönlichkeitsveränderungen. Als er sich schließlich im Boston Medical Center vorstellte, veranlassten Neurochirurgen um Dr. Nirav Patel eine umfangreiche Untersuchung.

\section{Riesige flüssigkeitsgefüllte Kugel}

Tatsächlich konnten sie eine deutliche Beeinträchtigung der Sehschärfe am rechten Auge feststellen, auch schnitt der Mann in neurokognitiven Tests für sein Alter überraschend schlecht ab. Daraufhin nahmen sie das Schädelinnere des Mannes per MRT sowie CT-Angiografie genauer unter die Lupe - und staunten nicht schlecht: Den vorderen Bereich füllte nicht Hirnmasse, sondern überwiegend eine flüssigkeitsgefüllte Kugel mit 70 mm Durchmesser. Sie drückte die Frontallappen zur Seite und nach hinten, wobei sie wohl auch den Sehnerv quetschte und zu Ödemen führte. Die kalzifizierte und partiell thrombosierte Hohlkugel erwies sich als ein Aneurysma der Arteria communicans anterior.

Die Ärzte um Patel veranlassten sofort einen Eingriff zur Reparatur des Aneurysmas und entfernten dabei auch die Thromben. Das linderte den Druck auf den Sehnerv und die Frontallappen, der Patient erholte sich nach dem Eingriff vollständig. Die Sehprobleme verschwanden ebenso wie die kognitiven Defizite nach einiger Zeit konnte er wieder seiner Arbeit nachgehen. Eine Nachuntersuchung zwei Jahre später zeigte, dass der Aneurysma-Sack komplett kollabiert war.

\section{0-fach erhöhtes Rupturrisiko}

Hirnaneurysmen mit einem Durchmesser von mehr als $25 \mathrm{~mm}$ sind sehr selten, schreiben die Neurochirurgen um Patel.
Unbehandelt ist das Rupturrisiko extrem hoch. Der Patient hatte also viel Glück, dass er nicht schon längst an einer Hirnblutung gestorben war. Weniger Glück hatte die Schwester des Mannes - sie war bereits an einer platzenden Gefäßaussackung im Gehirn verschieden. Schätzungsweise $20 \%$ der Patienten mit intrakraniellen Aneurysmen haben auch Verwandte ersten Grades mit einem solchen Problem, berichten die Ärzte aus Boston.

Nach Studiendaten ist bei einem Durchmesser von mehr als $20 \mathrm{~mm}$ das Risiko für eine Ruptur etwa 20-mal höher als bei einem Durchmesser unter $5 \mathrm{~mm}$. Die Gefahr einer Hirnblutung scheint mit dem Durchmesser der Aussackung exponentiell zu steigen.

Weitere bekannte Risikofaktoren für eine Ruptur sind Geschlecht, Alter und

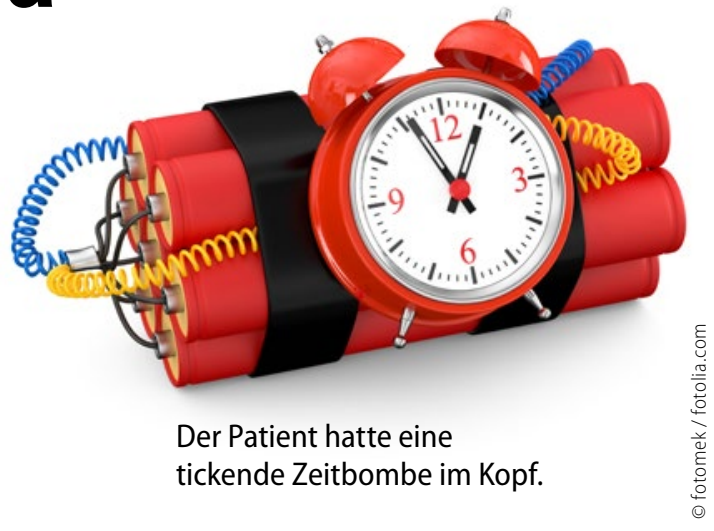

Nikotinkonsum. Bei Frauen und Rauchern ist das Lebenszeitrisiko für eine Ruptur im Vergleich zu Männern und Nichtrauchern in etwa verdoppelt, bei jungen Patienten (unter 50 Jahren) ist es etwa dreimal höher als bei älteren Betroffenen.

(Thomas Müller)

Patel NJ et al. A Giant Aneurysm of the Anterior Communicating Artery. N Engl J Med 2015; 373:560.

\section{Neues Positionspapier der GPA \\ Impfen macht nicht allergisch!}

Hilfreich könnte das neue Positionspapier u. a. im Umgang mit Eltern sein, die von diesem Umstand noch nicht so ganz überzeugt sind.

„Spezifische Standardimpfungen und Durchimpfung allgemein fördern nicht die allergische Sensibilisierung gegen Umweltallergene und fördern nicht die Entwicklung allergischer Erkrankungen wie Neurodermitis, Asthma und Heuschnupfen."

\section{Statements rund um's Impfen}

Dies ist nur eines von 16 Statements aus dem frisch aufgelegten Positionspapier der Gesellschaft Pädiatrischer Allergologie (GPA). Auch für die sich in Teilen der Bevölkerung hartnäckig haltende Vermu- tung, dass durchgemachte Infektionen das Allergierisiko senken könnten, gibt es keine Belege - so auch nicht im Zusammenhang mit Pertussis und Masern. Während Masern- und Keuchhustenimpfung keinerlei Effekte auf atopische Manifestationen hatten, waren durchgemachte Infektionen hier und da sogar mit einem erhöhten Allergierisiko assoziiert, berichtete Priv.-Doz. Dr. Christoph Grüber, Frankfurt, der maßgeblich an der Erarbeitung des Positionspapiers beteiligte Kinderarzt. Das Papier gibt zudem konkrete Informationen an die Hand, wie mit Kindern zu verfahren ist, die ein deutlich erhöhtes Allergierisiko mit sich tragen.

$(L R)$

Grüber C. Curriculum der Allergologie. 10. Deutscher Allergiekongress, Köln, 3. Oktober 2015 